\title{
Attoscience at ETH Zurich: Shining New Light on Old Questions in Quantum Mechanics
}

\author{
Ursula Keller*
}

\begin{abstract}
Ten years ago, we began a new research effort in attoscience at ETH Zurich, building on our ultrafast laser expertise in the few femtosecond regime. I present some of the technical highlights and explain how we continue within the NCCR MUST.
\end{abstract}

Keywords: Attosecond - Time-resolved spectroscopy · Tunneling time - Ultrafast science · Ultrafast solid-state lasers

Attoscience describes a new research field in time-resolved spectroscopy and in subfemtosecond lasers. Attosecond time resolution, where 1 attosecond (as) corresponds to $10^{-18}$ seconds, is required to resolve the dynamics of charge and energy transport on an atomic and molecular scale. These features are at the cutting edge of our NCCR MUST project, where the scientific driver is to better understand how matter functions at the electronic, atomic and molecular level; how matter changes its structure during a reaction; and how quanta of energy are transported on a microscopic spatial and ultrafast time scale. Attoscience is embedded in the MUST vision that we can contribute to important challenges such as alternative energy sources and improving health. We take a broader view to address these challenges through basic research, which we believe is fundamentally critical for breakthrough progress in these areas. My group started to work in attosecond science in 2001, mostly funded by a previous NCCR project in Quantum Photonics.

\section{'New Light'}

Continued progress in laser sources enables new discoveries and expands our knowledge horizon. For attoscience, the key enabling technology was the discovery of high harmonic generation (HHG), ${ }^{[1,2]}$ intense ultrafast near infrared pulses from Ti:sapphire laser systems, ${ }^{[3]}$ and carrier envelope offset (CEO) phase stabilization $^{[4-6]}$ with which single attosecond pulse generation first became possible. ${ }^{[7]}$ New demands on novel light sources also became apparent as we progressed in attoscience. One key limitation is the low pulse repetition rate from Ti:sapphire amplifier systems, which typically operate in the $1-\mathrm{kHz}$ regime, and generate attosecond pulses in the 1-nJ regime for harmonics up to about $100 \mathrm{eV}$. ${ }^{[8]}$ This means that we have at least a five orders of magnitude reduction in the signalto-noise ratio for attosecond pump probe measurements, compared to femtosecond sources which deliver similar pulse energies but at rates of about $100 \mathrm{MHz}$. However, just increasing the pulse energy does not usually solve this problem, as space charge limitations set an upper limit to the usable pulse energy. This has motivated us to continue our laser development in intense pulse generation but at megahertz repetition rates. ${ }^{[9]}$ High pulse repetition rate $f_{\text {rep }}$ of course then corresponds to an increase in average power $P_{a v}$, since $P_{a v}=$ $E_{p} f_{\text {rep }}$, where $E_{p}$ is the pulse energy. For example, a pulse energy of $100 \mu \mathrm{J}$ at a pulse repetition rate of $5 \mathrm{MHz}$ results in an average power of $500 \mathrm{~W}$. Such high average power is not practical for Ti:sapphire laser systems, and other solutions are currently being explored. Excellent thermal management is one key issue for average power scaling. Heat needs to be extracted from the laser gain medium, which is op- timized by a high surface-to-volume ratio of the gain medium as obtained in thin disk, fiber, or slab lasers, for example. Not surprisingly, all current world-leading results have been obtained with such lasers. In my group, we have focused on power scaling of SESAM modelocked thin disk lasers, which we first started in $2000,[10]$ and pushed in performance to above $10 \mu \mathrm{J}$ in $2008^{[11]}$ and currently to a record high average power of $140 \mathrm{~W}$ with an optical-to-optical pump efficiency of $40 \% .{ }^{[12]}$ In contrast to fiber ${ }^{[13]}$ and slab lasers, ${ }^{[14]}$ our results are generated directly from a laser oscillator - without any further external pulse amplification. Avoiding external amplifiers means that we avoid added noise from the amplifier and further system complexity. Our high power laser oscillators do not have any significantly higher complexity or more components than a standard low-power SESAM modelocked oscillator which is in any case required at the start of any amplifier system. Our goal is to explore the power scaling limitations of SESAM modelocked thin disk lasers in the 1-kW average output power regime, which we believe is reachable. At these power levels, an efficient, saturated power amplifier can always be added for further power scaling. A SESAM modelocked thin disk laser is ideally suited for power scaling because all optical elements inside the laser oscillator are used in reflection, laser mode areas can be increased as required, and SESAM damage can be avoided. ${ }^{[15]}$ In collaboration with Prof. Günter Huber's group in Hamburg, we are also exploring different laser materials to obtain shorter pulses, ${ }^{[16]}$ as we currently typically obtain around 800 -fs pulses at a center wavelength of around $1 \mu \mathrm{m}$, which then need to be compressed externally for attosecond pulse generation. ${ }^{[17,18]}$ 


\section{Attoline}

Since 2001 my group has invested a significant amount of time and resources in developing an 'attoline' (Fig. 1) for attosecond pulse generation and attosecond spectroscopy. In the first attoline generation, we were able to explore very fundamental processes in HHG. We explored attosecond pulse train (APT) assisted HHG, ${ }^{[21,22]}$ which promises a significant efficiency enhancement. Typical HHG efficiencies at present are in the $10^{-6}$ to $10^{-8}$ regime. For a clear experimental demonstration however, we required higher energy from our Ti:sapphire laser system and improved mechanical stability for a spatially separated attosecond delay line. Solving these issues were not required, however, for the first observation of the theoretically predicted quantum path interference (QPI) in HHG. ${ }^{[23-26]}$ The simple three step model in HHG predicted QPI because we have two or more electron trajectories contributing to harmonic emission within the plateau harmonics, i.e. the short and long trajectory, which results in an intensity-dependent phase difference introduced by the classical excursion time of the excited electrons in the strong laser field before recombination and harmonic emission. Phase matching and angular apertures allowed us to balance the different contributions from the short and long trajectories such that the interference contrast was sufficient for observation in HHG.

Even though we could obtain many interesting results with our first attoline, it became apparent that significant limitations in mechanical stability did not allow for stable attosecond pulse characterization and for a larger variety of attosecond measurements. Our next generation attoline (Fig. 1) was mainly designed and constructed by two graduate students Mirko Holler[19] and Florian Schapper[20] starting at the beginning of 2007. The much improved mechanical stability allowed us to measure attosecond pulses for the first time in May 2008 ${ }^{[19,20]}$ using the RABBITT technique ${ }^{[27]}$ (Fig. 2).

\section{Attosecond Transient Absorption}

Attosecond transient absorption is an all-optical method for time-resolved measurements in the attosecond domain and has been widely used in the femtosecond domain. An all-optical method has several advantages compared to photoelectron detection as shown for example in Fig. 2. They are less affected by space charge effects, exhibit a much better signal-to-noise ratio and a higher dynamic range, and allow for much faster acquisition times of minutes instead of hours. We could demonstrate attosecond control of absorption of different harmonics where a moderately strong infrared pulse introduces rapid absorption modulations. The relative phase of these oscillations in the individual harmonics can be controlled by the infrared pulse intensity. ${ }^{[29]}$ This control can be explained by the interference of transiently bound electron wave packets as every pulse in the attosecond pulse train (APT) creates a transiently bound electronic wavepacket. Two consecutive attosecond pulses within the APT create two electrons wave packets: the first one returns to the ion after its laser driven trajectory and then interferes with the newly created second one. The phase delay of this trajectory is intensity dependent and therefore the interference and recombination is also intensity dependent. These process is repeated with half-cycle periodicity. This experiment represents the first all-optical observation of electron wavepacket interference on attosecond timescale.
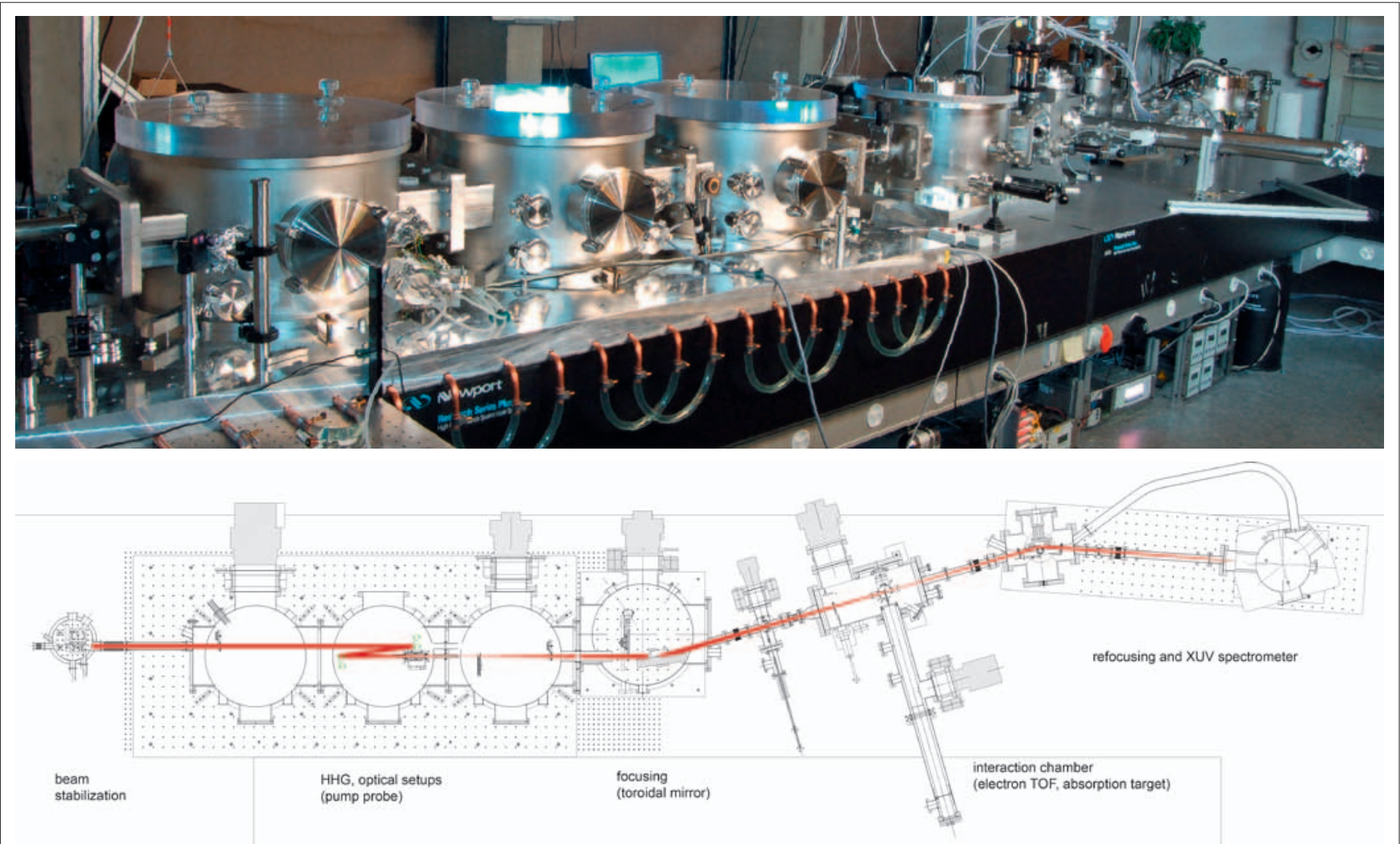

Fig. 1. Attoline at ETH Zurich. ${ }^{[19,20]}$ From left to right: The first three identical chambers accommodate the high-harmonics generation target and an interferometer for pump-probe-like experiments. The next chamber contains a toroidal focusing mirror directing the VUV-XUV and infrared beams into the experimental target chamber. This chamber is equipped with a time-of-flight (TOF) spectrometer. On the most right-hand side, an XUV spectrometer is attached for photon diagnostics. Note that the high power infrared laser amplifier system is not shown here and comes from the left into the first chamber for HHG. 


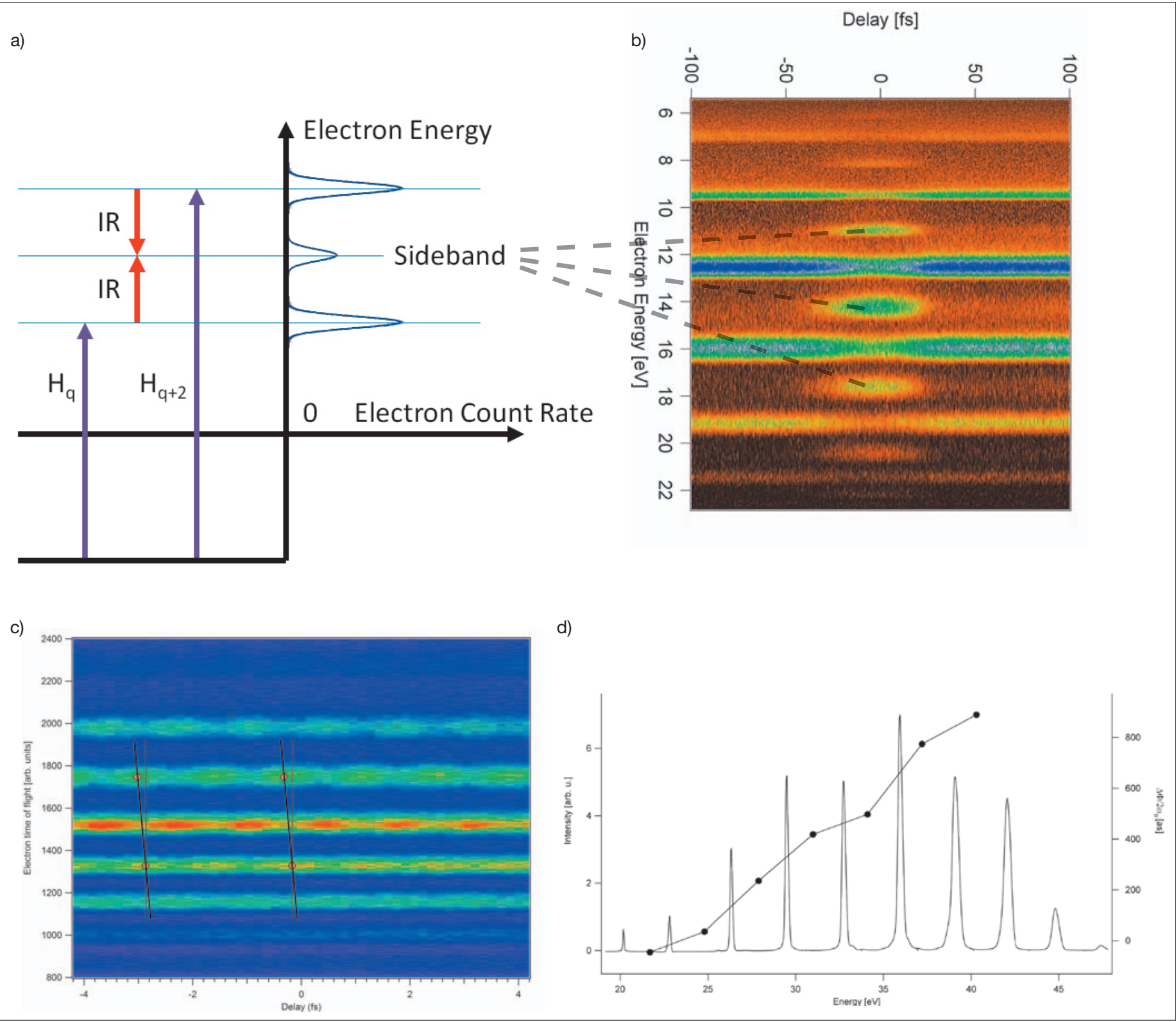

Fig. 2. Characterization of an attosecond pulse train (APT) $)^{[19,20]}$ with the RABBITT technique ${ }^{[27]}$ which determines the phase difference between two adjacent harmonics (top left). For the electron time-of-flight detection (top right) delay steps of 107 as (i.e. $15 \mathrm{~nm}$ steps controlled with a piezo) with an acquisition time per step of $60 \mathrm{~s}$ have been applied. For a full RABBITT trace (bottom left) stable conditions for more than 30 hours are required. The recorded phase difference (bottom right) then determines the attochirp of the APT. Here we measured 450 as for the average pulse duration of the attosecond pulses within the APT with a transform limit of 160 as. With an additional 500-nm thick Al filter for dispersion compensation close to transform limited pulses can be achieved. ${ }^{[28]}$

\section{Attoclock}

The attoclock ${ }^{[30,31]}$ is a novel attosecond streaking technique and allows for unprecedented time resolution in the attosecond regime. The attoclock principle is based on an intense, close-to-circular polarized laser field, where the rotating electric field vector gives the time reference similar to the hands of a clock. The attoclock has been used to measure the tunneling delay time in strong laser field ionization, which is defined by the angular difference between the maximum of the electric laser field, which induces the highest tunneling ionization rate, and the direction of the laser field when the electron exits the tunnel and can be accelerated by the electric field vector direction at that exact moment at the exit of the tunnel. This measurement is based on the definition of 'time' by 'counting cycles' where the rotating electric laser field vector defines the cycle and where we count fractions of a cycle. The short laser pulse limits the ionization event within one single optical cycle, which is about $2.7 \mathrm{fs}$ at a center wavelength of $800 \mathrm{~nm}$. A temporal resolution of only a few attoseconds can be achieved because the measurement is based on an angular 'peak search' within one optical cycle. The observables are the final momentum of the electron and ion in coincidence with much less than one ionization event per laser pulse. The final distribution is averaged over many ionization events, which give a certain angular probability distribution in ionization. The final momentum of ion and electron can be measured in coincidence using a COLTRIMS (COLd Target Recoil Ion Momentum Spectroscopy $)^{[32]}$ setup and which we constructed with the help of Prof. Reinhard Dörner's group from Frankfurt University. We measured an instantaneous tunneling delay time in helium in the low intensity regime, ${ }^{[31]}$ within a measurement accuracy of around 10 attoseconds.

We then used the attoclock technique to ask fundamental questions regarding how fast a strong laser field can remove two electrons from an atom and if there are any correlation effects between these two electrons. ${ }^{[33,34]}$ This has been studied with argon for a strong laser field process 
referred to as double ionization. In strong field double ionization the standard picture typically distinguishes between sequential double ionization (SDI), where the electrons are assumed to tunnel-ionize independently, and non-sequential double ionization (NSDI), where the electrons cannot be treated separately. Electron correlation in double ionization by linearly polarized laser pulses has been studied extensively, both theoretically and experimentally. The question about electron correlation in double ionization by circularly polarized laser pulses is currently a hot topic in many theoretical studies. Electron correlation in strong field double ionization is dominated by recollision of the first ionized electron with its parent ion. With laser pulses that are close to circularly polarized, recollision may be greatly modified or avoided and the electrons are usually assumed to be field ionized without mutual interaction. An open question remained whether other mechanisms rather than recollision can occur in strong field double ionization. Elliptically (close to circularly) polarized pulses are ideally suited to answer this question, since recollision of the first emitted electron with the parent ion is prevented. Moreover with close to circularly polarized pulses we can apply the attoclock technique and determine the ionization time of the two electrons. The magnitude of the electron momenta follows the envelope of the laser pulse and gives a coarse timing for the electron releases (i.e. 'the hour hand of the attoclock'). The emission angle of the electrons subsequently gives the fine timing (i.e. 'the minute hand of the attoclock'). We have found unexpected results: First, coincidence momentum data exhibit an intensity dependence that is not captured by the standard SDI model, as shown with an oscillatory intensity dependence for the ratio of parallel to antiparallel electron emission. ${ }^{[34]}$ Second, the release time of the second ionized electron occurs significantly earlier than predicted by an SDI model. ${ }^{[33]}$

\section{Old Questions in Quantum Mechanics}

Novel time-resolved attosecond streaking techniques such as energy streaking ${ }^{[35]}$ and the attoclock (i.e. angular streaking) ${ }^{[31]}$ are currently being applied in an attempt to answer very fundamental questions in quantum mechanics: How fast can light remove a bound electron or even many electrons from an atom or a solid? How fast can energy and charge be transported within a molecule, or from a surface to an adsorbed molecule? How fast is tunneling? Tunneling is one of the most fundamental concepts in quantum mechanics and is of fundamental importance for energy and charge transport in technology and nature.

For example two different attosecond measurement techniques addressed two different but related fundamental processes: strong laser field ionization, where the strong laser field bends the binding potential to emit an electron by tunneling (tunnel ionization $)^{[31]}$ and the photoelectric effect, where a single light quantum (a photon) is absorbed to emit an electron (photoemission). ${ }^{[36]}$ In the first case, an instantaneous tunneling delay time in helium was measured within the experimental accuracy of a few tens of attoseconds, and in the latter case, a relative delay in photoemission of about 20 as between electrons originating from different bound states in neon was measured - from a semi-classical point of view a somewhat puzzling outcome! Strong laser field ionization involves the absorption of many photons, and furthermore the electron emitted into the continuum (i.e. an unbound state) initially experiences a spatial separation from the ion of more than ten atomic units. For photoemission, in contrast, only one photon is absorbed without any initial lateral displacement. Why does photoemission, in contrast to tunnel ionization, have a measurable emission delay, we may wonder? As we know, quantum mechanics may give unexpected results as viewed from a classical perspective. Future results should hopefully help to resolve this potential controversy and give us a better physical picture to understand attosecond quantum mechanics.

More recently, we were able to add further insight. [37] After our first attoclock experiments published in December 2008, we continued to perform laser tunnel ionization experiments on both atomic helium and argon with attosecond time resolution over a larger intensity range, covering both the multiphoton ionization and the tunnel ionization regime. This work required very careful experimental efforts to prevent any artifacts and the theoretical support from the group of Prof. Lars Madsen at Aarhus University to resolve our initially unexpected results. By comparing the helium and argon results, we could show that a tunneling model correctly describes the data, assuming instantaneous tunneling delay time within the measurement accuracy of around a few 10 attoseconds. However tunneling occurs through a potential barrier that is modified by all of the remaining electrons and from a state that is shifted significantly by the external field. The resulting modified force terms influence the dynamics of the tunneled electron, changes important physical parameters and hence need to be accounted for in attosecond measurement techniques described above. The multi- electron effects and the corrections to the tunnel potential identified in this work for the first time are universal in all atomic and molecular systems. These results affect attosecond streaking measurements because additional force terms need to be considered that have not been considered before. Leaving them away would result in a wrong delay time!

Our future work within MUST will greatly benefit from this new insight and will make sure that such attosecond streaking measurements are construed very carefully, since these multi-electron effects will become even more severe for molecules and surfaces, which will be a focus of our NCCR MUST projects. For a single atom the multi-electron dynamics were so fast that the static response was sufficient to explain our experimental results. It will be interesting to explore more complex and potentially slower multi-electron dynamics in molecules and surfaces. We are still at the beginning of a long and interesting journey with potentially many unexpected outcomes.

\section{Acknowledgements}

Many excellent graduate students and postdocs have made essential contributions since I have joined the physics faculty at ETH Zurich in March 1993. I also would like to acknowledge the financial support from ETH Zurich and the Swiss National Science Foundation (SNSF) within the NCCR Quantum Photonics that allowed me to start a new research focus in attoscience in 2001 and the great introduction to high field laser physics I received in Prof. Anne L'Huillier's group at the Lund University in Sweden during my sabbatical in 2001.

Received: March 6, 2011

[1] M. Ferray, A. L'Huillier, X. F. Li, L. A. Lompré, G. Mainfray, C. Manus, J. Phys. B: At. Mol. Opt. Phys. 1988, 21, L31.

[2] A. McPherson, G. Gibson, H. Jara, U. Johann, T. S. Luk, I. A. McIntyre, K. Boyer, C. K. Rhodes, J. Opt. Soc. Am. B 1987, 4, 595.

[3] P. F. Moulton, J. Opt. Soc. Am. B 1986, 3, 125.

[4] H. R. Telle, G. Steinmeyer, A. E. Dunlop, J. Stenger, D. H. Sutter, U. Keller, Appl. Phys. B 1999, 69, 327

[5] A. Apolonski, A. Poppe, G. Tempea, C. Spielmann, T. Udem, R. Holzwarth, T. W. Hänsch, F. Krausz, Phys. Rev. Lett. 2000, 85, 740.

[6] D. J. Jones, S. A. Diddams, J. K. Ranka, A Stentz, R. S. Windeler, J. L. Hall, S. T. Cundiff, Science 2000, 288, 635.

[7] M. Drescher, M. Hentschel, R. Kienberger, M. Uiberacker, V. Yakovlev, A. Scrinzi, T. Westerwalbesloh, U. Kleineberg, U. Heinzmann, F. Krausz, Nature 2002, 419, 803.

[8] U. Keller, IEEE Photonics Journal 2010, 2, April.

[9] T. Südmeyer, S. V. Marchese, S. Hashimoto, C. R. E. Baer, G. Gingras, B. Witzel, U. Keller, Nature Photonics 2008, 2, 599.

[10] J. Aus der Au, G. J. Spühler, T. Südmeyer, R. Paschotta, R. Hövel, M. Moser, S. Erhard, M. Karszewski, A. Giesen, U. Keller, Opt. Lett. 2000, 25, 859 . 
[11] S. V. Marchese, C. R. E. Baer, A. G. Engqvist, S. Hashimoto, D. J. H. C. Maas, M. Golling, T. Südmeyer, U. Keller, Opt. Express 2008, 16, 6397.

[12] C. R. E. Baer, C. Kränkel, C. J. Saraceno, O. H Heckl, M. Golling, R. Peters, K. Petermann, T. Südmeyer, G. Huber, U. Keller, Opt. Lett. 2010 , 35, 2302.

[13] T. Eidam, S. Hanf, E. Seise, T. V. Andersen, T. Gabler, C. Wirth, T. Schreiber, J. Limpert, A. Tünnermann, Opt. Lett. 2010, 35, 94.

[14] P. Russbueldt, T. Mans, J. Weitenberg, H. D. Hoffmann, R. Poprawe, Opt. Lett. 2010, 34, 4169.

[15] C. J. Saraceno, C. Schriber, M. Mangold, M. Hoffmann, O. H. Heckl, C. R. E. Baer, M. Golling, T. Südmeyer, U. Keller, IEEE J. Sel. Top. Quantum Electron. 2011, DOI: 10/1109/ JSTQE.2010.2092753.

[16] T. Südmeyer, C. Kränkel, C. R. E. Baer, O. H. Heckl, C. J. Saraceno, M. Golling, R. Peters, K. Petermann, G. Huber, U. Keller, Appl. Phys. B 2009, 97, 281.

[17] T. Südmeyer, F. Brunner, E. Innerhofer, R. Paschotta, K. Furusawa, J. C. Baggett, T. M. Monro, D. J. Richardson, U. Keller, Opt. Lett. 2003, 28, 1951.

[18] C. J. Saraceno, O. H. Heckl, C. R. E. Baer, T. Südmeyer, U. Keller, Opt. Express 2011, 19, 1395.

[19] M. Holler, Ph.D. thesis, ETH Zurich Nr. 18782 , 2009.

[20] F. Schapper, Ph.D. thesis, ETH Zurich Nr. 18783, 2009.

[21] K. J. Schafer, M. B. Gaarde, A. Heinrich, J. Biegert, U. Keller, Phys. Rev. Lett. 2004, 92, 023003.

[22] M. B. Gaarde, K. J. Schafer, A. Heinrich, J. Biegert, U. Keller, Phys. Rev. A 2005, 72, 013411.

[23] A. Zaïr, M. Holler, A. Guandalini, F. Schapper, J. Biegert, L. Gallmann, U. Keller, A. S. Wyatt, A. Monmayrant, I. A. Walmsley, E. Cormier, T. Auguste, J. P. Caumes, P. Salières, Phys. Rev. Lett. 2008, 100, 143902.

[24] T. Auguste, P. Salières, A. S. Wyatt, A. Monmayrant, I. A. Walmsley, E. Cormier, A. Zaïr, M. Holler, A. Guandalini, F. Schapper, J. Biegert, L. Gallmann, U. Keller, Phys. Rev. A 2009, 80, 033817.
[25] M. Holler, A. Zaïr, F. Schapper, T. Auguste, E. Cormier, A. Wyatt, A. Monmayrant, I. A. Walmsley, L. Gallmann, P. Salières, U. Keller, Opt. Express 2009, 17, 5716.

[26] F. Schapper, M. Holler, T. Auguste, A. Zair, M. Weger, J. P. Caumes, P. Salieres, L. Gallmann, U. Keller, Opt. Express 2010, 18, 2987.

[27] P. M. Paul, E. S. Toma, P. Breger, G. Mullot, F. Augé, P. Balcou, H. G. Muller, P. Agostini, Science 2001, 292, 1689.

[28] R. Lopez-Martens, K. Varju, P. Johnsson, J. Mauritsson, Y. Mairesse, P. Salieres, M. B. Gaarde, K. J. Schafer, A. Persson, S. Svanberg, C.-G. Wahlstrom, A. L'Huillier, Phys. Rev. Lett. 2005, 94, 033001.

[29] M. Holler, F. Schapper, L. Gallmann, U. Keller, Phys. Rev. Lett. 2011, 106, 123601.

[30] P. Eckle, M. Smolarski, P. Schlup, J. Biegert, A. Staudte, M. Schöffler, H. G. Muller, R. Dörner, U. Keller, Nat. Phys. 2008, 4, 565.

[31] P. Eckle, A. Pfeiffer, C. Cirelli, A. Staudte, R. Dörner, H. G. Muller, M. Büttiker, U. Keller, Science 2008, 322, 1525.

[32] R. Dörner, V. Mergel, O. Jagutzki, L. Spielberger, J. Ullrich, R. Moshammer, H. Schmidt-Bocking, Phys. Rep. 2000, 330, 95.

[33] A. N. Pfeiffer, C. Cirelli, M. Smolarski, R. Dörner, U. Keller, Nat. Phys. 2011, 7, 428; K. Ueda, K. L. Ishikawa, Nat. Phys. 2011, 7, 371.

[34] A. N. Pfeiffer, C. Cirelli, M. Smolarski, R. Dorner, U. Keller, 'Breakdown of the independent electron approximation in sequential double ionization', submitted.

[35] R. Kienberger, E. Goulielmakis, M. Uiberacker, A. Baltuska, V. Yakovlev, U. Heinzmann, M. Drescher, F. Krausz, Nature 2004, 427, 817.

[36] M. Schultze, M. Fiess, N. Karpowicz, J. Gagnon, M. Korbman, M. Hofstetter, S. Neppl, A. L. Cavalieri, Y. Komninos, T. Mercouris, C. A. Nicolaides, R. Pazourek, S. Nagele, J. Feist, J. Burgdorfer, A. M. Azzeer, R. Ernstorfer, R. Kienberger, U. Kleineberg, E. Goulielmakis, F. Krausz, V. S. Yakovlev, Science 2010, 328, 1658.

[37] A. N. Pfeiffer, C. Cirelli, M. Smolarski, D. Dimitrovski, M. Abu-Samha, L. B. Madsen, U. Keller, 'Attoclock reveals geometry of laserinduced tunnel ionization', http://arxiv.org/ $a b s / 1103.4803 v 2$. 